\title{
Obtaining the biomechanical behavior of ascending aortic aneurysm via the use of novel speckle tracking echocardiography
}

\author{
Mohammed Alreshidan, MD, MSc(C), ${ }^{\text {a,d }}$ Nastaran Shahmansouri, MSc, ${ }^{c}$ Jennifer Chung, MD, MSc, ${ }^{a}$ \\ Vynka Lash, FRCPC, ${ }^{\mathrm{b}}$ Alexander Emmott, MEng, ${ }^{\mathrm{c}}$ Richard L. Leask, PhD, ${ }^{\mathrm{c}}$ and \\ Kevin Lachapelle, MD, FACS, FRCSC ${ }^{\mathrm{a}}$
}

\begin{abstract}
Introduction: Ex vivo measurement of ascending aortic biomechanical properties may help understand the risk for rupture or dissection of dilated ascending aortas. A validated in vivo method that can predict aortic biomechanics does not exist. Speckle tracking transesophageal echocardiography (TEE) has been used to measure ventricular stiffness; we sought to determine whether speckle TEE could be adapted to estimate aortic stiffness in vivo and compare these findings with those obtained by ex vivo tissue measurements.
\end{abstract}

Methods: A total of 17 patients undergoing ascending aortic resection were recruited to with a mean aortic diameter was $56.16 \pm 15 \mathrm{~mm}$. Intraoperative speckle TEE tracking analysis was used to calculate aortic stiffness index using the following equation: $\beta 2=\ln (S B P / D B P) / A o S$, where $\beta 2$ is the stiffness index; SBP is systolic blood pressure; DBP is diastolic blood pressure; and AoS is the circumferential strain. Ex vivo stiffness was obtained by mechanical tissue testing according to previously described methods. The aortic ring at the pulmonary trunk was divided into 4 equal quadrants.

Results: The in vivo stiffness index for the inner curvature, anterior wall, outer curvature, and posterior wall were $0.0544 \pm 0.0490,0.0295 \pm 0.0199$, $0.0411 \pm 0.0328$, and $0.0502 \pm 0.0320$, respectively. The mean ex vivo $25 \%$ apparent stiffness for inner curvature, anterior wall, outer curvature, and posterior wall were $0.0616 \pm 0.0758 \mathrm{MPa}, 0.0352 \pm 0.00992 \mathrm{MPa}, 0.0405 \pm 0.0199 \mathrm{MPa}$, and $0.0327 \pm 0.0106 \mathrm{MPa}$, respectively. The patient-matched ex vivo $25 \%$ apparent stiffness and in vivo stiffness index were not significantly different ( $P=.8617,2$-way analysis of variance with repeated measures).

Conclusions: The use of speckle TEE appears to be a promising technique to estimate ex vivo mechanical properties of the ascending aortic tissue. (J Thorac Cardiovasc Surg 2017;153:781-8)

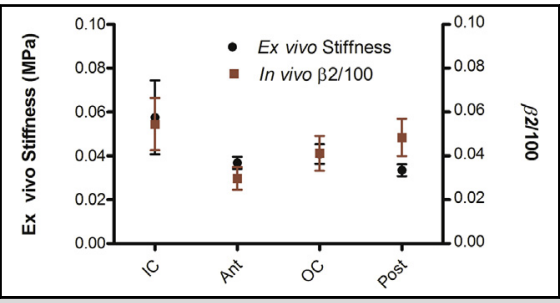

In vivo stiffness index versus ex vivo stiffness (no statistical difference, $P=.8617$ ).

\section{Central Message}

Transesophageal echocardiography speckle tracking may be used to assess mechanical behavior of ascending aorta; further modeling and increased number of patients will be required to better highlight high-risk patients.

\section{Perspective}

We believe that biomechanics is a promising tool to assess and predict aortic aneurysm complications; coupling transesophageal echocardiography and blood pressure monitoring has allowed us to obtain the biomechanical behavior of ascending aorta and this may help in predicting aortic complications in high-risk patients, and hence planning for management.

See Editorial Commentary page 789.

See Editorial page 779 .
An ascending aortic diameter greater than $5.5 \mathrm{~cm}$ is the main indication for aortic resection to manage the increased

\footnotetext{
From the Departments of ${ }^{\mathrm{a} C a r d i a c}$ Surgery, ${ }^{\mathrm{b}}$ Anesthesia, and ${ }^{\mathrm{c}} \mathrm{Chemical}$ Engineering, McGill University, Montreal, Quebec, Canada; and ${ }^{\mathrm{d}}$ King Salman Heart Center, King Fahad Medical City, Riyadh, Saudi Arabia.

Read at the 96th Annual Meeting of The American Association for Thoracic Surgery, May 14-18, 2016, Baltimore, Maryland.

Received for publication June 6, 2016; revisions received Oct 11, 2016; accepted for publication Nov 4, 2016; available ahead of print Jan 13, 2017

Address for reprints: Kevin Lachapelle, MD, FACS, FRCSC, Cardiac Surgery Department, Royal Victoria Hospital, McGill University Health Center - Glen Site, 1001 Boul. Decarie, C07 1284, Montreal, Quebec H3A 3J1, Canada (E-mail: kevin.lachapelle@mcgill.ca).

$0022-5223 / \$ 36.00$

Copyright $(2016$ by The American Association for Thoracic Surgery http://dx.doi.org/10.1016/j.jtcvs.2016.11.056
}

risk of rupture or dissection. ${ }^{1-4}$ There is epidemiologic evidence to suggest that there is a hinge point at $5.5 \mathrm{~cm}$ where the risk of aortic complication is increased markedly. $^{2-4}$ This hinge point also is supported by the study of biomechanical properties of ex vivo aortic tissue. $^{5-7}$ Tissue remodeling and medial degeneration occurs with increased diameter of aorta, resulting in loss

Scanning this QR code will take you to a supplmental video. To view the AATS 2016 Webcast, see the URL next to the video thumbnail.

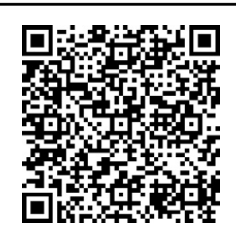




$$
\begin{aligned}
& \text { Abbreviations and Acronyms } \\
& \begin{aligned}
\text { ANOVA } & =\text { analysis of variance } \\
\text { AoS } & =\text { circumferential strain } \\
\beta 2 & =\text { in vivo stiffness index } \\
\text { LV } & =\text { left ventricle } \\
\text { TEE } & =\text { transesophageal echocardiography } \\
\text { 2D } & =2 \text {-dimensional }
\end{aligned}
\end{aligned}
$$

of the elastic properties and increased stiffness. ${ }^{8-11}$ Moreover, aortic tissue remodeling has been noted as part of the aging process and is seen in patients with connective tissue disease and aortic aneurysms. It is this remodeling process that may make the aorta at risk for rupture or dissection.

Our focus was to try to determine whether the biomechanics estimated in vivo can eventually help determine the risk of complications in patients with tissue remodeling by comparing the results with ex vivo mechanical analysis and histopathology. Stiffness is a measure of the resistance offered by tissue to an applied force and can be calculated from the stress-strain relationship of the tissue via the use of a tensile tester. ${ }^{5,6,12,13}$ Our group, along with others in the field, have found that the biomechanics of aortic tissue may be a good predictor of the histological integrity of the aortic wall. ${ }^{9,11,14}$ Biomechanics, and therefore the integrity of the aortic wall, may be a better marker of risk than size alone, especially given the observation that most dissections occur in aortas smaller than $5.5 \mathrm{~cm} .{ }^{15}$ The major limitation to the use of biomechanics in risk prediction is the absence of a noninvasive method to measure the biomechanical properties of the aorta in vivo.

Recently, there has been an interest in estimating biomechanics in vivo by calculating tissue strain via echocardiography and magnetic resonance. ${ }^{16-22}$ Speckle tracking is an echocardiographic imaging technique that can measure the motion of cardiac tissue by using the naturally occurring speckle patterns in tissue or blood created by ultrasound frequencies during cardiac imaging. The motion of the speckles during systole and diastole allows one to calculate strain. Much work has been done using speckle tracking to calculate left ventricle (LV) strain. ${ }^{23-25}$ The stress on the tissue can be estimated by recording the pressure during the cardiac cycle.

We hypothesized that transesophageal echocardiography (TEE) using speckle tracking, as is performed on the LV, could be used to measure the deformation of the ascending aorta during the cardiac cycle. This, coupled with the blood pressure, would allow us to estimate the in vivo stiffness index and compare these values with the ex vivo biomechanical properties. This is a first step to determine whether echocardiography speckle tracking can be used to better stratify patients for ascending aortic replacement surgery.

\section{METHODS}

\section{Patient Population}

Patients undergoing ascending aortic resection were recruited to be part of the study. Patients with acute aortic dissection and connective tissue disease such as Marfan syndrome were excluded from the cohort. Patients who could not undergo a transesophageal study also were excluded. All ascending aortic samples were obtained from operating room at the time of surgery at McGill University Health Center in Montreal, Canada, following informed consent.

\section{Intraoperative TEE and In Vivo Speckle Tracking Strain Imaging Analysis}

The TEE study was performed with a Vivid 7, GE echo machine (GE Healthcare, Madison, Wis) with simultaneous electrocardiogram tracking as routine preparation for the operation. After the patient was anesthetized, the TEE probe was inserted into the esophagus to the level of great vessels to obtain the short-axis view of ascending aorta at the level of the pulmonary trunk (Figure 1). The measurements are taken with the patient off all inotropes/vasopressors and normotensive with a heart rate in the normal range. The blood pressure was recorded simultaneously by the use of radial artery line tracing. In preparation for echo, a Swan-Ganz catheter was pulled back and the ventilation turned off to minimize the lung movement during capturing of the loop of the ascending aorta. A short-axis view of the ascending aorta at the level of maximum dilatation or at the level of pulmonary artery was recorded by using the 2-dimensional (2D) echo image. The image was recorded during 5 heartbeats and the view optimized to guarantee that all walls of ascending aorta were visualized. A mid-esophagus $120^{\circ}$ long-axis view of ascending aorta also was obtained. The blood pressure during the study was recorded.

The speckle echo tracking analysis was done for all ascending aortic loops postoperatively with the GE EchoPAC station (GE Healthcare). Strain mapping of the circumference of the aorta in short axis was done using advanced Q Analysis, 2D strain. Between 14 and 18 markers (depending on the aortic size) were set to demark the inner wall of the aorta. Figure 2 is an explanatory illustration showing the setup for the echo speckle tracking analysis. Video 1 demonstrates the way we set and calculate the circumferential strain. The minimum circumferential length of the aorta during the heartbeat was identified and set as L0. This length was used as a base to define the 4 quadrants of the ascending aorta (inner curvature, anterior wall, outer curvature, posterior wall). The length of each region was assumed to be equal. The software tracked the changes in the length of the aorta with time during the heartbeat. The final length $L_{f}$ and the

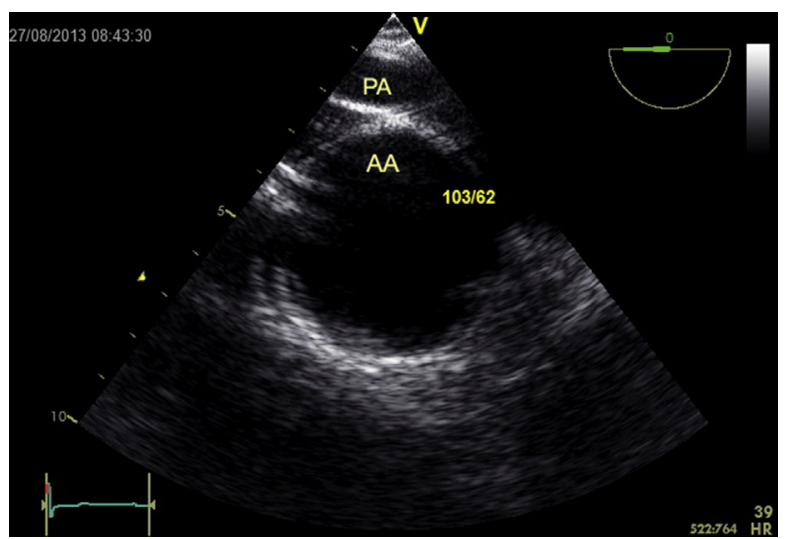

FIGURE 1. Short-axis view of the ascending aorta using TEE at the level of maximal dilatation or the pulmonary artery. Blood pressure $103 / 39 \mathrm{~mm}$ $\mathrm{Hg}$. $P A$, pulmonary artery; $V$, position of probe; $A A$, ascending aorta. 


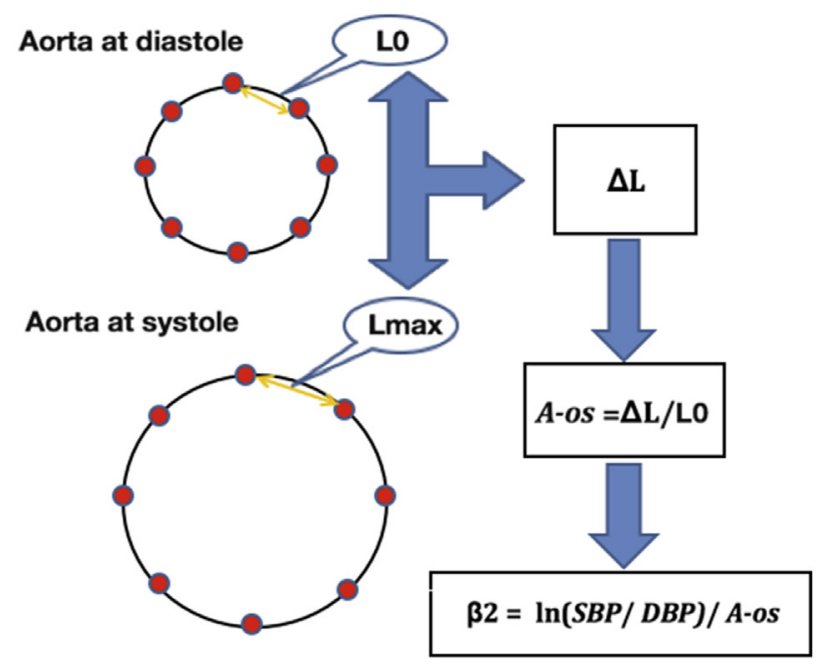

A

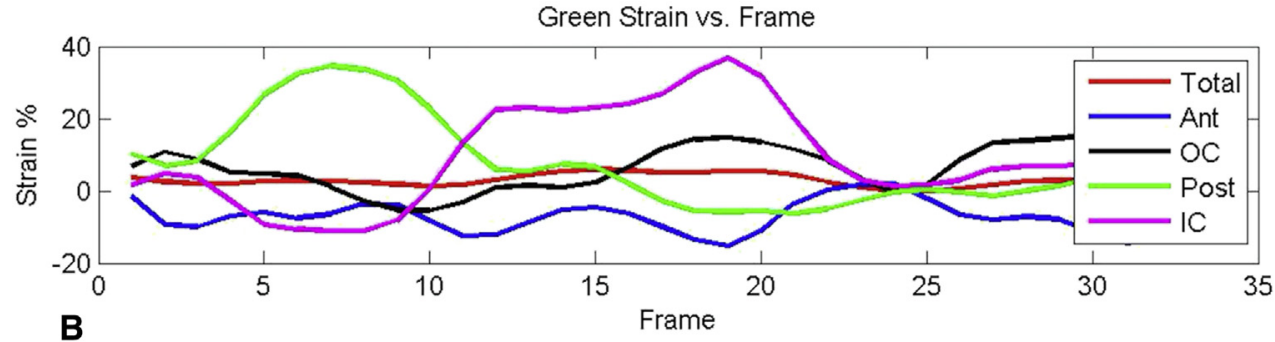

FIGURE 2. A, Illustration of the in vivo echo strain calculation. B, Echo-calculated strain mapping for each region of the aorta. $L O$, Original length; $\Delta L$, the length difference; Lmax, maximum length; $A o S$, circumferential strain; $\beta 2$, in vivo stiffness index; $S B P$, systolic blood pressure; $D B P$, diastolic blood pressure.

original length $L_{o}$ of each quadrant during the heartbeat were used to calculate the green strain, which we took as the AoS for each region.

Green strain $\in_{\text {Green }}=A o S=\frac{1}{2}\left(\frac{L_{f}^{2}-L_{o}^{2}}{L_{o}^{2}}\right)\left(L_{f}:\right.$ final length; $L_{o}$ : original length)

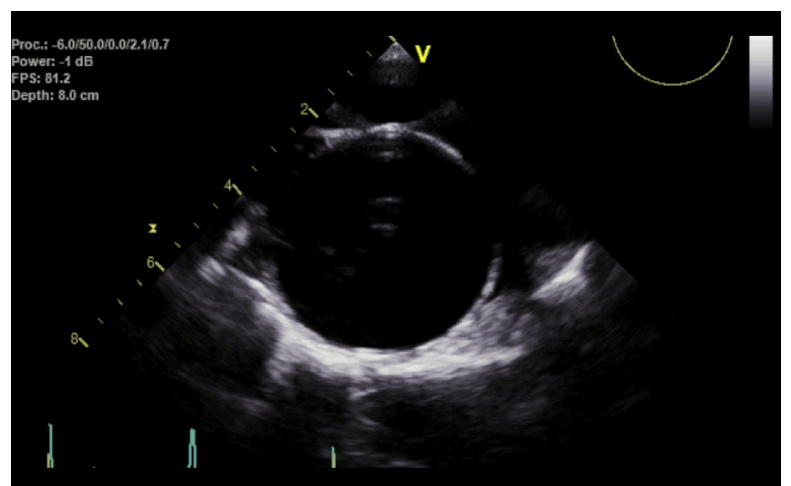

VIDEO 1. TEE speckle tracking strain mapping for 61-year-old man with bicuspid aortic valve and ascending aortic aneurysm, the ascending aorta was captured above the sinuo-tubular junction and at the level of pulmonary artery trunk. Video available at: http://www.jtcvsonline.org/article/ S0022-5223(16)31680-4/addons.
To obtain the stiffness index from echo calculation, we used the empirical formula defined by Hirai and colleagues ${ }^{26}$ and adapted for $2 \mathrm{D}$ echocardiographic strain imaging by Oishi and colleagues. ${ }^{21}$ Originally, Hirai and colleagues ${ }^{26}$ fit a semilogarithmic equation to intraluminal pressures and the external diameter of the human abdominal aorta by defining the distension ratio $(\lambda)$ as the arterial diameter (D1 at systolic pressure) at a given pressure (P systolic), normalized by the diameter (Do at diastolic pressure) at a standard pressure (P diastole). When they plotted the distention ratio against the logarithmic value of the relative pressure, a linear relation was observed in the physiologic range of pressure. ${ }^{26}$

$\ln (S B P / D B P)=\beta 2(($ diameter at $\mathrm{SBP}-$ diameter at $\mathrm{DBP}) /$ diameter at DBP). ${ }^{26}$ Oishi and colleagues ${ }^{21}$ adapted this metric for $2 \mathrm{D}$ echocardiography-derived strain and defined the stiffness index as:

$\beta 2=\ln (S B P / D B P) / A o S$, where $\beta 2$ is the stiffness index; SBP is the systolic blood pressure; DBP is the diastolic blood pressure; and AoS is the circumferential strain.

For our analysis, we defined the AoS as the maximum green strain, $\epsilon_{\text {Green }}$ for each segment of the aorta.

\section{Tissue Preparation and Ex Vivo Aortic Stiffness}

The tissue was obtained from the operating room as an intact ring with a single clip marker at the anterior wall of the aneurysm belly. ${ }^{9}$ The tissue was kept in ice until we performed the mechanical testing. The mechanical testing was done within 24 hours from tissue collection. Sections of $1.5 \times 1.5 \mathrm{~cm}$ were collected from each quadrant of the aortic wall (inner curvature, anterior, outer curvature, and posterior). Each square underwent equibiaxial tensile testing at $37^{\circ} \mathrm{C}$ in Ringer's lactate solution. After preconditioning, 3 cycles at $0.01 \mathrm{~mm} / \mathrm{s}$ were used to capture the tissue 
TABLE 1. Patient demographic data

\begin{tabular}{lc}
\hline \multicolumn{1}{c}{ Variable } & Mean \pm SD or $\mathbf{~}$ \\
\hline Sex (male) & $64(11 / 17)$ \\
Age, y & $63.9 \pm 15.9$ \\
Ejection fraction & $60 \pm 10$ \\
Hypertension & $47(8 / 17)$ \\
Diabetes mellitus & $5.8(1 / 17)$ \\
Dyslipidemia & $23(4 / 17)$ \\
Aortic diameter, mm & $56.16 \pm 15$ \\
Bicuspid aortic valve & $41(7 / 17)$ \\
Aortic stenosis & $47(8 / 17)$ \\
Aortic valve replacement & $88(15 / 17)$ \\
Bentall procedure & $64(11 / 17)$ \\
David valve-sparing operation & $5.8(1 / 17)$ \\
Calcium channel blocker & $41(7 / 17)$ \\
Beta blocker & $53(9 / 17)$ \\
\hline
\end{tabular}

$S D$, Standard deviation

stress-strain relationship (EnduraTEC Electro Force 3200 Biaxial Tensile Tester; Bose Corporation, Eden Prairie, Minn).

The analysis of mechanical data was done with Matlab R2012a (MathWorks, Natick, Mass). We calculated the green strain, $\in_{\text {Green }}$, based on the displacement of the sutures securing the tissue during the biaxial loading. These data, along with the load applied and cross-sectional area of the tissue, were used to obtain the stress-strain curves, where the stress was defined as the second Piola-Kirchhoff stress:

$S=\frac{F L_{o}}{A_{o} L}=\left(F:\right.$ load; $L_{o}:$ original length; $L$ : final length; $A_{o}$ : initial crosssectional area).

The apparent modulus of elasticity (slope of the average stress-strain curve) was calculated at $25 \%$ green strain, which approximates the physiological condition. ${ }^{27}$

\section{Statistical Analysis}

The statistical analysis was performed with GraphPad Prism 5 (GraphPad Software Inc, San Diego, Calif). A difference in the means was considered significant for a $P$ value was less than .05 . The regional variation between the ex vivo and in vivo strain index analysis was compared with a 2-way analysis of variance (ANOVA) and a

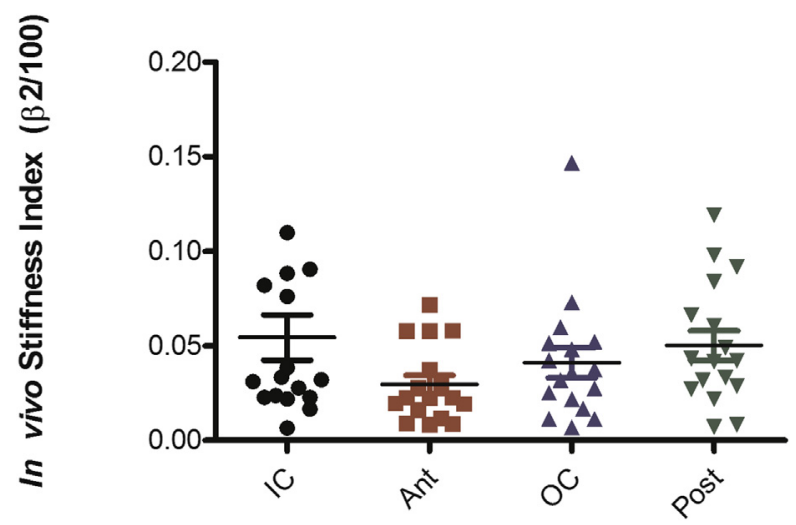

FIGURE 3. In vivo-estimated stiffness index mean and standard deviation for all patients. IC, Inner curvature; Ant, anterior wall, $O C$, outer curvature; Post, posterior wall.
Bonferroni multiple comparison test. One-way ANOVAs were done to evaluate the regional variation of both the ex vivo apparent stiffness and in vivo stiffness index estimates.

\section{RESULTS}

Table 1 summarizes the demographics data for the patients involved in the study. Fifty-seven percent of the patients were male and the mean aortic diameter of all patients was $56.16 \pm 15 \mathrm{~mm}$. The mean ejection fraction was $60 \pm 10 \%$, and $42 \%$ of the patients were hypertensive. Furthermore, approximately one third of the patients had a bicuspid aortic valve (36\%), and $42 \%$ of the total cohort had aortic stenosis. In terms of the blood pressure medication, $36 \%$ of the patients used calcium channel blockers and $47 \%$ were on beta-blockers.

The estimated in vivo stiffness index was scaled by dividing it by 100 to be similar in magnitude to the ex vivo apparent modulus expressed in MPa. For the in vivo stiffness index obtained by TEE, the average $\beta 2 /$ 100 , for the inner curvature, anterior wall, outer curvature, and posterior wall were $0.0544 \pm 0.0490$, $0.0295 \pm 0.0199,0.0411 \pm 0.0328$, and $0.0502 \pm 0.0320$, respectively. The anterior wall had the lowest stiffness index and was significantly less than the inner curvature and posterior wall $(P=.01$ and $P=.05$, respectively, Bonferroni multiple comparison test), Figure 3.

For the ex vivo stiffness as measured by tissue biomechanics, there was a similar pattern of apparent stiffness as in vivo samples, with the outer curvature the stiffest; however, there was much less variation in the data. The mean ex vivo $25 \%$ apparent stiffness for inner curvature, anterior wall, outer curvature, and posterior wall was $0.0616 \pm 0.0758 \mathrm{MPa}, 0.0352 \pm 0.00992 \mathrm{MPa}$, $0.0405 \pm 0.0199 \mathrm{MPa}$, and $0.0327 \pm 0.0106 \mathrm{MPa}$, respectively, Figure 4.

A 2-way ANOVA was used to evaluate whether there was a significant difference between the mean values of the ex vivo stiffness and in vivo stiffness index, and if the results were dependent on region. The patient matched ex vivo $25 \%$ apparent stiffness and in vivo stiffness index $(\beta 2 /$ $100)$ were not significantly different $(P=.8617,2$-way ANOVA with repeated measures). The results were dependent on location $(P=.0372,2$-way ANOVA with repeated measures), Figure 5. A pairwise post Bonferonni multiple comparison test showed no significant difference between in vivo or ex vivo measurements in any of the regions $(P>.05$, post- Bonferonni multiple comparison test); however, there was no significant linear correlation between the in vivo and ex vivo stiffness $\left(\mathrm{R}^{2}=0.158, P=.1137\right)$.

\section{DISCUSSION}

Our main goal in this study was to determine whether the speckle tracking performed from TEE data could be used to estimate the biomechanical features of the ascending aorta. 


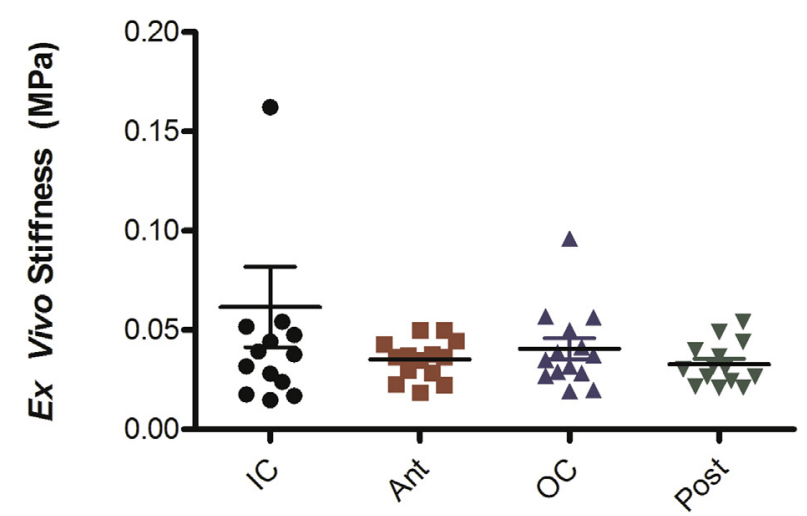

FIGURE 4. Ex vivo-calculated stiffness mean and standard deviation for all patients. $I C$, Inner curvature; Ant, anterior wall, $O C$, outer curvature; Post, posterior wall.

To our knowledge, this is one of the first studies of its kind comparing estimates of a stiffness index obtained in vivo during TEE to those obtained ex vivo on the same aortic tissue. This is a pilot study to determine whether echocardiography speckle tracking can reliably estimate the biomechanical tissue properties of aneurysmal tissue in our patient population. This is the first step in validating whether such measures correlate with the degree of tissue remodeling and ultimately help to better stratify patients for ascending aortic replacement surgery. We found no significant difference in the mean values obtained by the 2 methods. The results suggest there is some merit in the in vivo stiffness index estimate as a measure of aortic tissue biomechanics, but much more work is needed to make this method a reliable and predictable clinical tool.

Speckle tracking echo, was used initially to calculate the LV strain and deformation. ${ }^{28}$ Speckle-tracking echo detects the deformation of tissue such as the LV and allows one to estimate the deformation in the 3 different directions, longitudinal, radial, and circumferential. ${ }^{28}$ The circumferential strain of the LV has been defined as shortening of LV

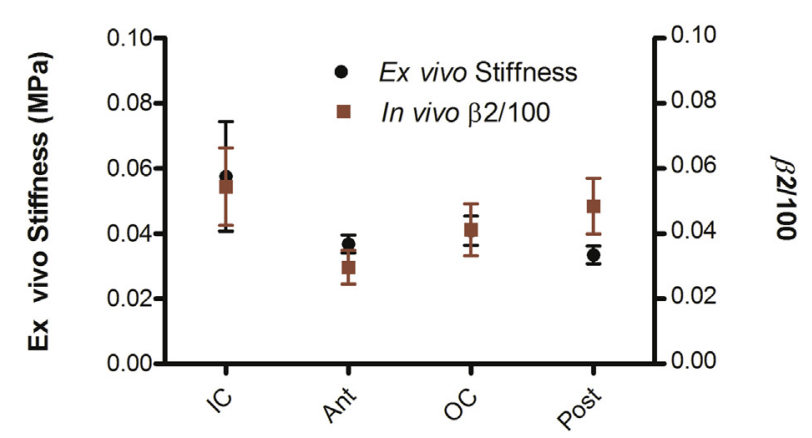

FIGURE 5. Ex vivo stiffness and in vivo stiffness index $(\beta / 100)$ regional wall variation. $I C$, Inner curvature; $A n t$, anterior wall, $O C$, outer curvature; Post, posterior wall; $\beta 2$, in vivo stiffness index. myocardial fiber along the circular perimeter observed in a short-axis view. ${ }^{28}$

To calculate the in vivo stiffness index, we adapted the methods used to calculate the stiffness index in the abdominal aorta by Oishi and colleagues. ${ }^{21}$ In their work, Oshi and colleagues used similar equipment and the entire circumference to define the peak AoS over 3 cardiac cycles and compared the results with M-mode ultrasonographic estimates of stiffness as defined by Hirai and colleagues. ${ }^{26}$ Oishi and colleagues ${ }^{21}$ showed $\beta 2$ stiffness index to increase with age and increased dramatically with advanced age ( $>50$ years). In normal individuals, they found the stiffness index to be linearly related to age.

Our results showed no correlation of in vivo stiffness index or ex vivo apparent stiffness with age (data not shown). This finding is not surprising, because our patient population was exclusively aneurysmal and at risk of rupture and dissection. The cohort presented by Oishi and colleagues ${ }^{21}$ was of consecutive patients undergoing routine health checkups.

We adjusted our in vivo stiffness index by a factor of 100 to allow for similar magnitude as our ex vivo apparent stiffness expressed in MPa. The in vivo stiffness index as derived by Hirai and colleagues ${ }^{26}$ is dimensionless. The ex vivo apparent stiffness depends on the units of stiffness, in our case, MPa. Because of the nonlinear nature of human aortic tissue biomechanics, the apparent stiffness changes with the level of strain. In our study, we have chosen to use $25 \%$ green strain, which is representative of our in vivo strain values.

The average in vivo stiffness index for all our patients was $4.3 \pm 3.0$, which is lower than the values found by Oishi and colleagues ${ }^{21}$ for the abdominal aorta. Oishi and colleagues $^{21}$ found in patients younger than 50 years old the average stiffness index was $8 \pm 4$, whereas patients older than 50 years had a stiffness index $29 \pm 20$. The difference between the abdominal and ascending aorta stiffness index and our ascending aorta values are attributable to the structure of the aorta, which changes along its length. It has been shown that the aorta becomes less elastic with increasing distance from the heart. ${ }^{29-31}$ The variation in mechanical properties along the aorta is partly the result of changes in the tissue composition. The elastin content decreases along the length of the aorta. ${ }^{32}$

The in vivo calculated stiffness index showed a significant difference between anterior, inner curvature, and posterior wall (Figure 3). The ex vivo calculations showed a similar but nonsignificant trend. The difference in the in vivo stiffness index may be explained partially by the anatomical differences when we compared the anterior wall with the inner curvature and posterior wall. The posterior wall and inner curvature of the aorta are supported by various structures such as pulmonary artery, left atrium, and the trachea, limiting the motion of these parts of the 
aortic wall. Epi-aortic ultrasound has shown the movement in the anterior and posterior aneurysmal ascending aortic wall to be limited. ${ }^{33}$

It is expected that in vivo measurements would have more variability and potentially less precision than the controlled ex vivo data. First, in vivo evaluation is effected by the surrounding structures such as trachea, left atrium, and pulmonary artery, which can affected the strain values, especially in the posterior and inner curvature, as demonstrated by Modak and colleagues ${ }^{33}$ during motion evaluation of the aortic wall using epi-aortic ultrasound. In addition, patient blood pressure was not controlled. This overall leads to greater variability in the in vivo data. In contrast, ex vivo mechanical testing is performed on an area of aortic tissue without the effect of the soundings anatomical structures or the whole intact aorta. Second, the calculation methodology was different between the 2 modalities. The ex vivo values are based on the direct measurement of the cyclical stress-strain relationship of the tissue under controlled mechanical loading. The stiffness index/100 is an empirical fit to stiffness data under physiological conditions ${ }^{21}$ and therefore not directly representative of our ex vivo conditions. The in vivo stiffness index is not the same mathematical definition as the apparent stiffness. We have artificially adjusted our in vivo estimate to be of similar magnitude as the ex vivo measured apparent stiffness. We have estimated, based on literature values, a representative in vivo $\operatorname{strain}^{27}$ and used the stress-strain relationship to calculate the ex vivo stiffness. Because the mechanical stress-strain curve of ascending aortic tissue is nonlinear, ${ }^{5,6,9}$ selecting one arbitrary strain to estimate the in vivo stiffness index is very simplistic. Furthermore, the in vivo estimated stiffness index depends mainly on the blood pressure of the patient and varies between patients. Despite these simplifications, we found good agreement between the in vivo stiffness index $(\beta 2 / 100)$ and the $25 \%$ ex vivo apparent modulus (Figure 5). Paired analysis between the in vivo and ex vivo data show the stiffness index $\beta 2$ is similar to ex vivo values. More work needs to be done to improve the variability of the in vivo stiffness index and to investigate more-reliable metrics of aortic biomechanics.

Ex vivo-measured mechanical properties have shown very good correlations with histological changes that occur with ascending aortic aneurysms. ${ }^{9}$ Our group found the energy loss to be a more robust mechanical metric for predicting aortic remodeling than stiffness. ${ }^{9}$ The energy loss is calculated from the integral of the stress-strain curve and uses the full-cycle stress-strain data, as opposed to the stiffness, which is described at one point in the curve. The strain mapping from 2D echocardiograms provide the full cardiac cycle strain data, which could be combined with the aortic pressure waveform. In fact, we have now started to collect in vivo strain data throughout the cardiac cycle, allowing us to construct a more complete stress-strain curve for each aorta. This will be more precise then a single estimate of the strain-stress relationship, which is what was performed in this study. Moreover, we will move forward to compare transthoracic echo aortic strain with TEE speckle tracking and mechanical data to truly make this a noninvasive and widely available method.

This study is limited by a relatively small sample size. Moreover, we have grouped both bicuspid and tricuspid valves and have no normal tissue controls. Our in vivo measurements had much more variability then our ex vivo measures, and this may reflect operator technique or selection of speckle points. Furthermore, this program originally was constructed to be used in the LV strain calculation, and we used it to calculate the strain in the aortic wall. Issues with the view of the aorta and interference of surrounding tissue might be overcome by dedicated software and routines for the ascending aorta. Although the results show promise for in vivo estimates of ascending aorta biomechanics, from a practical point of view, the methodology is not ready to be applied to clinical decision making.

\section{CONCLUSIONS}

The use of speckle tracking performed during TEE appears to be a promising technique to estimate ex vivo mechanical properties of the ascending aortic tissue. In this study, mean stiffness values obtained in vivo were similar to those measured ex vivo. More work is needed to make TEE-derived strain mapping a reliable method for stratifying ascending aortic aneurysm patients for surgery.

\section{Webcast}

You can watch a Webcast of this AATS meeting presentation by going to: http://webcast.aats.org/2016/Video/Monday/0516-16_Room_340_1800_Alreshidan-800.mp4.

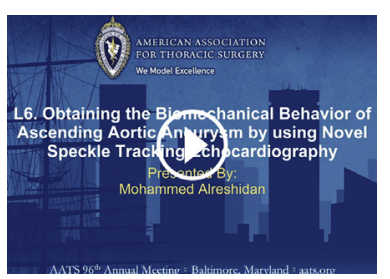

\section{Conflicts of Interest Statement}

Authors have nothing to disclose with regard to commercial support.

\section{References}

1. Coady MA, Rizzo JA, Hammond GL, Kopf GS, Elefteriades JA. Surgical intervention criteria for thoracic aortic aneurysms: a study of growth rates and complications. Ann Thorac Surg. 1999;67:1922-6; discussion 1953-8.

2. David TE. Surgical treatment of ascending aorta and aortic root aneurysms. Prog Cardiovasc Dis. 2010;52:438-44. 
3. Elefteriades JA. Indications for aortic replacement. J Thorac Cardiovasc Surg. 2010;140(6 suppl):S5-9; discussion S45-51.

4. Hiratzka LF, Bakris GL, Beckman JA, Bersin RM, Carr VF, Casey DE, et al. 2010 ACCF/AHA/AATS/ACR/ASA/SCA/SCAI/SIR/STS/SVM guidelines for the diagnosis and management of patients with Thoracic Aortic Disease: a report of the American College of Cardiology Foundation/American Heart Association Task Force on Practice Guidelines, American Association for Thoracic Surgery, American College of Radiology, American Stroke Association, Society of Cardiovascular Anesthesiologists, Society for Cardiovascular Angiography and Interventions, Society of Interventional Radiology, Society of Thoracic Surgeons, and Society for Vascular Medicine. Circulation. 2010;121:e266-369.

5. Choudhury N, Bouchot O, Rouleau L, Tremblay D, Cartier R, Butany J, et al. Local mechanical and structural properties of healthy and diseased human ascending aorta tissue. Cardiovasc Pathol. 2009;18:83-91.

6. Okamoto RJ, Wagenseil JE, DeLong WR, Peterson SJ, Kouchoukos NT, Sundt TM. Mechanical properties of dilated human ascending aorta. Ann Biomed Eng. 2002;30:624-35.

7. Okamoto RJ, Xu H, Kouchoukos NT, Moon MR, Sundt TM. The influence of mechanical properties on wall stress and distensibility of the dilated ascending aorta. J Thorac Cardiovasc Surg. 2003;126:842-50.

8. Carlson RG, Lillehei CW, Edwards JE. Cystic medial necrosis of the ascending aorta in relation to age and hypertension. Am J Cardiol. 1970;25:411-5.

9. Chung J, Lachapelle K, Wener E, Cartier R, De Varennes B, Fraser R, Leask RL. Energy loss, a novel biomechanical parameter, correlates with aortic aneurysm size and histopathologic findings. J Thorac Cardiovasc Surg. 2014;148: 1082-8; discussion 1088-9.

10. El-Hamamsy I, Yacoub MH. Cellular and molecular mechanisms of thoracic aortic aneurysms. Nat Rev Cardiol. 2009;6:771-86.

11. Schlatmann TJ, Becker AE. Histologic changes in the normal aging aorta: implications for dissecting aortic aneurysm. Am J Cardiol. 1977;39:13-20.

12. Khanafer K, Duprey A, Zainal M, Schlicht M, Williams D, Berguer R. Determination of the elastic modulus of ascending thoracic aortic aneurysm at different ranges of pressure using uniaxial tensile testing. J Thorac Cardiovasc Surg. 2011; 142:682-6.

13. Tremblay D, Zigras T, Cartier R, Leduc L, Butany J, Mongrain R, Leask RL. A comparison of mechanical properties of materials used in aortic arch reconstruction. Ann Thorac Surg. 2009;88:1484-91.

14. Pisano C, Maresi E, Balistreri CR, Candore G, Merlo D, Fattouch K, et al. Histological and genetic studies in patients with bicuspid aortic valve and ascending aorta complications. Interact Cardiovasc Thorac Surg. 2012;14:300-6.

15. Pape LA, Tsai TT, Isselbacher EM, Oh JK, Gara PTO, Evangelista A, et al. Aortic diameter $>$ or $=5.5 \mathrm{~cm}$ is not a good predictor of type A aortic dissection: observations from the International Registry of Acute Aortic Dissection (IRAD). Circulation. 2007;116:1120-7.

16. Allen BD, Barker AJ, Carr JC, Silverberg RA, Markl M. Time-resolved threedimensional phase contrast MRI evaluation of bicuspid aortic valve and coarctation of the aorta. Eur Heart J Cardiovasc Imaging. 2013;14:399.

17. Burk J, Blanke P, Stankovic Z, Barker A, Russe M, Geiger J, et al. Evaluation of 3D blood flow patterns and wall shear stress in the normal and dilated thoracic aorta using flow-sensitive 4D CMR. J Cardiovasc Magn Reson. 2012;14:84.

18. Firmin DN, Nayler GL, Kilner PJ, Longmore DB. The application of phase shifts in NMR for flow measurement. Magn Reson Med. 1990;14:230-41.

19. Imura T, Yamamoto K, Kanamori K, Mikami T, Yasuda H. Non-invasive ultrasonic measurement of the elastic properties of the human abdominal aorta. Cardiovasc Res. 1986;20:208-14.

20. Markl M, Wallis W, Harloff A. Reproducibility of flow and wall shear stress analysis using flow-sensitive four-dimensional MRI. J Magn Reson Imaging. 2011;33:988-94.

21. Oishi Y, Miyoshi H, Mizuguchi Y, Iuchi A, Nagase N, Oki T. Aortic stiffness is strikingly increased with age $>/=50$ years in clinically normal individuals and preclinical patients with cardiovascular risk factors: assessment by the new technique of 2D strain echocardiography. J Cardiol. 2011;57:354-9.

22. Yamashita S, Isoda H, Hirano M, Takeda H, Inagawa S, Takehara Y, et al. Visualization of hemodynamics in intracranial arteries using time-resolved threedimensional phase-contrast MRI. J Magn Reson Imaging. 2007;25:473-8.

23. Voigt JU, Pedrizzetti G, Lysyansky P, Marwick TH, Houle H, Baumann R, et al. Definitions for a common standard for 2D speckle tracking echocardiography: consensus document of the EACVI/ASE/Industry Task Force to standardize deformation imaging. J Am Soc Echocardiogr. 2015;28:183-93.

24. Marwick TH, Leano R, Brown J, Sun J-P, Hoffmann R, Lysyansky P, et al. Myocardial strain measurement with 2-dimensional speckle-tracking echocardiography: definition of normal range. JACC Cardiovasc Imaging. 2009;2:80-4.
25. Levy PT, Machefsky A, Sanchez AA, Patel MD, Rogal S, Fowler S, et al. Reference ranges of left ventricular strain measures by twodimensional speckle-tracking echocardiography in children: a systematic review and meta-analysis. J Am Soc Echocardiogr. 2016;29:209-25.e6.

26. Hirai T, Sasayama S, Kawasaki T, Yagi S. Stiffness of systemic arteries in patients with myocardial infarction. A noninvasive method to predict severity of coronary atherosclerosis. Circulation. 1989;80:78-86.

27. Duprey A, Khanafer K, Schlicht M, Avril S, Williams D, Berguer R. In vitro characterisation of physiological and maximum elastic modulus of ascending thoracic aortic aneurysms using uniaxial tensile testing. Eur J Vasc Endovasc Surg. 2010;39:700-7

28. Mondillo S, Galderisi M, Mele D, Cameli M, Lomoriello VS, Zacà V, et al Speckle-tracking echocardiography: a new technique for assessing myocardial function. J Ultrasound Med. 2011;30:71-83.

29. Learoyd BM, Taylor MG. Alterations with age in the viscoelastic properties of human arterial walls. Circ Res. 1966;18:278-92.

30. Han HC, Fung YC. Longitudinal strain of canine and porcine aortas. J Biomech 1995;28:637-41

31. Rouleau L, Tremblay D, Cartier R, Mongrain R, Leask RL. Regional variations in canine descending aortic tissue mechanical properties change with formalin fixation. Cardiovasc Pathol. 2012;21:390-7.

32. Fry P, Harkness MLR, Harkness RD, Nightingale M. Mechanical properties of tissues of lathyritic animals. J Physiol. 1962;164:77-89.

33. Modak RK, Koullias GJ, Govindarajulu US, Tranquilli M, Barash PG Elefteriades JA. Ascending aortic aneurysms: asymmetrical differences in aortic cross-sectional wall motion detected by epiaortic echocardiography. J Cardiothorac Vasc Anesth. 2010;24:776-9.

Key Words: aorta, aneurysm, risk of rupture, echo speckle tracing, biomechanics

\section{Discussion}

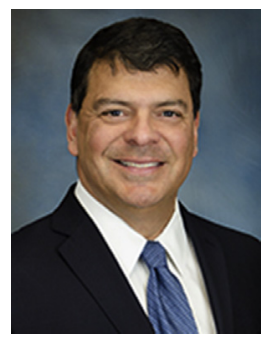

Dr A. DeAnda (Galveston, Tex). Thank you for the opportunity to discuss this paper, Mohammed. Thank you for sending me the manuscript in advance as well.

What you're describing here has very good translational significance. As aortic surgeons, we're always wondering when is the right time to operate? Who is at risk for progression, dissection, or rupture? How should these patients be followed - do you follow someone with serial computed tomography scans or magnetic resonance imaging (MRI)? And, ideally, you want to operate on a dissection the day before it happens, but we can't always do that.

You don't really discuss it in your slides here but you mention it in the manuscript, and my first question is, you show no relationship between the age of the patient and stiffness, which is counter-intuitive because we do know that the aortic stiffness changes as you get older due to the loss of elastin and changes in smooth muscle in the wall of the aorta.

Why do you think you did not see any difference or relationship between age and stiffness in your study? 


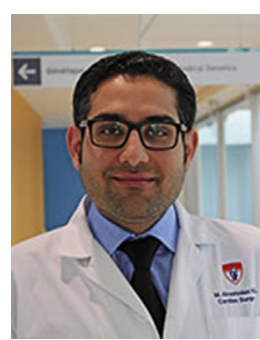

Dr Alreshidan. Thank you very much, Dr DeAnda, for reviewing the manuscript. This is a very good question actually.

As you know, in this study we just looked into 19 patients, and all 19 patients have similar pathology which is an ascending aortic aneurysm.

So in this group, you could find the patient who had a bicuspid aortic valve who is in his 40s, and he had a very severe dilatation of his aorta. And the stiffness actually is high compared with the patient who was in his 70s with a tricuspid valve whose aorta is actually less dilated and less stiff.

So, I mean, I didn't find a significant correlation giving that point, and also because the group of the patients is very small, just including 19 patients.

Dr DeAnda. You also, and you do mention it in your talk, excluded connective tissue disorders which I find interesting and maybe because it was a limitation on your patient population, but I think that would have been interesting because these patients tend to have problems at a smaller diameter. And I would suspect that you would still see the changes in your stiffness but at a smaller diameter.

Dr Alreshidan. Thank you also for that question. Actually, we have limited patients with connective tissue disease coming to our institution. That's one point.

The second point, as you know, Marfan disease, their problem is mainly to their aortic root other than their ascending aorta. And we keep in mind that using the patient with connective tissue disease, it may affect the results and skew the data. So for that reason, just for the sake of this study, we just make our population as homogenous as we can by using only the patients with ascending aortic aneurysm without any connective tissue disease.

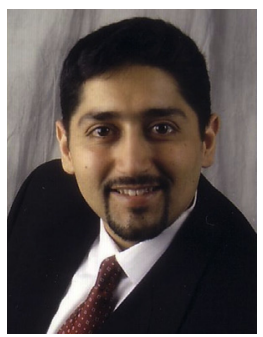

Dr O. Al-Radi (Jeddah, Saudi Arabia). Thank you, Mohammed. I enjoyed your presentation.

Could you apply the same principles to MRI, which have better spatial resolution and are less invasive than a TEE and still give you full cardiac cycle measurement of the aorta?

Dr Alreshidan. Thank you, Dr Al-Radi, for this question. Actually in our group, now we start doing some MRI for these same patients. MRI is a very good modality for obtaining the biomechanics of the aorta. It's well known and it's not only to give you the mechanics of the aorta but to give you the flow rate of the aorta and will also be able to calculate the wall shear stress. And you can also develop a model in predicting the growth of the ascending aortic aneurysm. But we choose to use echo because echo is easier to obtain. It's less expensive, and it's easy to apply everywhere because accessibility to MRI is not very well in all places. But, of course, MRI is a very good modality. 